PROCEEDINGS OF THE

AMERICAN MATHEMATICAL SOCIETY

Volume 130, Number 5, Pages 1401-1406

S 0002-9939(01)06288-8

Article electronically published on October 12, 2001

\title{
THE EXTENSION OF POSITIVE DEFINITE OPERATOR-VALUED FUNCTIONS DEFINED ON A SYMMETRIC INTERVAL OF AN ORDERED GROUP
}

\author{
MIHÁLY BAKONYI
}

(Communicated by Joseph A. Ball)

\begin{abstract}
Let $G_{1}$ be an ordered abelian group and $a \in G_{1}$. Let $G_{2}$ be an abelian group and $f$ an operator-valued positive definite function on $(-a, a) \times$ $G_{2}$. We prove that $f$ admits a positive definite extension to $G_{1} \times G_{2}$, generalizing in this way existing results for the case when $G_{1}=\mathbf{R}$ and $f$ is continuous.
\end{abstract}

\section{INTRODUCTION}

Let $G$ be an abelian group and let $\Lambda$ be a finite subset of $G$. A function $k: S=\Lambda-\Lambda \rightarrow \mathcal{L}(\mathcal{H})$ (the algebra of bounded linear operators on a Hilbert space $\mathcal{H}$ ) is called positive (semi)definite with respect to $\Lambda$ if, for every finite subset $\lambda_{1}, \lambda_{2}, \cdots, \lambda_{n} \in \Lambda$, the operator matrix $\left\{k\left(\lambda_{i}-\lambda_{j}\right)\right\}_{i, j=1}^{n}$ is positive (semi)definite. Without loss of generality, we assume in this paper that positive definite functions $k$ have the property that $k(0)=I_{\mathcal{H}}$. Let $G_{1}$ be an ordered abelian group, $a \in G_{1}$, and $G_{2}$ be an abelian group. A function $k:(-a, a) \times G_{2} \rightarrow \mathcal{L}(\mathcal{H})$ is referred to as positive definite if it is positive definite with respect to $[0, a) \times G_{2}$, unless otherwise specified. M. G. Krein proved [10] that every positive definite continuous scalar function on a real interval $(-a, a)$ admits a continuous positive definite extension to R. A. P. Artjomenko [2] (see also Theorem 4.2.3 in [15]) provided a new proof for Krein's Extension Theorem without the continuity requirement. Y. M. Berezansky and I. M. Gali ([6], see also Theorem 5.4.4.2 in [5]) proved the following extension of Krein's Theorem: "Given a Hilbert space $\mathcal{H}$, and a positive definite function $k$ on a layer in $\mathcal{H}$ that is $J$ continuous at 0 , then $k$ can be extended to a positive definite function on $\mathcal{H}$ with the same property of continuity." By a similar proof it follows that every continuous positive definite function on $(-a, a) \times G$, where $G$ is a topological group, can be extended to a continuous positive definite function on $\mathbf{R} \times G$.

J. Friedrich and L. Klotz [9] proved that, given $0<a<\infty$ and a topological group $G$, any strongly continuous positive definite function $k:(-a, a) \times G \rightarrow \mathcal{L}(\mathcal{H})$ admits a positive definite extension to $\mathbf{R} \times G$. The aim of this paper is to generalize the above result by omitting the continuity requirement and by substituting $\mathbf{R}$ with an ordered abelian group. Finally, several corollaries of our main result are presented. One of them is a result recently proved in [7, and the others are

Received by the editors November 14, 2000.

1991 Mathematics Subject Classification. Primary 43A35, 47A57, 42A70, 47A20.

(C)2001 American Mathematical Society 
generalizations of extension results for positive definite functions in [16], [13], and [4].

For notation and results in group theory and Fourier Analysis on groups we refer to [14] and [15]. If $G$ is a locally compact abelian group, then its character group is denoted by $\Gamma$.

Suppose $P$ is a semigroup of the abelian group $G$ and that $P$ has the properties

$$
P \cap(-P)=\{0\}, \quad P \cup(-P)=G .
$$

Under these conditions, $P$ induces an order in $G$. If we define $x \geq y$ to mean $x-y \in P$, then the axioms of linear order are satisfied. The choice of a semigroup $P$ with the above properties makes $G$ into an ordered group.

Let $K$ be a compact Hausdorff space and let $\mathcal{B}(K)$ denote the class of Borel measurable sets in $K$ and $C(K)$ the set of all continuous complex functions on $K$. A function $F: \mathcal{B}(K) \rightarrow \mathcal{L}(\mathcal{H})$ such that $F(X)=I_{\mathcal{H}}$ is called a semispectral measure if for every $h \in \mathcal{H}, \mu(\sigma)=(F(\sigma) h, h)$ is a positive Borel measure on $K$. If $F$ is a semispectral measure, define the Borel measures $\mu_{h, k}(\sigma)=(F(\sigma) h, k)$ for $\sigma \in \mathcal{B}(\mathcal{H})$ and $h, k \in \mathcal{H} ;\left\{\mu_{h, k}\right\}_{h, k \in \mathcal{H}}$ is called the semispectral family associated with $F$. The following results are well-known facts (see, e.g., Theorem 7.1 and Proposition 9.2 in [17).

Theorem 1.1. Let $X$ be a compact Hausdorff space and let $L: C(K) \rightarrow \mathcal{L}(\mathcal{H})$ be a linear operator such that $L(1)=I_{\mathcal{H}}$. Then $L$ is positive in the sense that $L(q) \geq 0$ for every $q \in C(K)$ if and only if there exists a semispectral family $\left\{\mu_{h, k}\right\}_{h, k \in \mathcal{H}}$ on $K$ such that

$$
(L(q) h, k)=\int_{K} q(x) d \mu_{h, k}(x)
$$

for every $q \in C(K)$ and $h, k \in \mathcal{H}$.

Theorem 1.2. Let $G$ be an abelian group. A function $f: G \rightarrow \mathcal{L}(\mathcal{H})$ with $f(0)=$ $I_{\mathcal{H}}$ is positive definite if and only if there exists a semispectral family $\left\{\mu_{h, k}\right\}_{h, k \in \mathcal{H}}$ on $\Gamma$ such that

$$
(f(x) h, k)=\int_{\Gamma} \gamma(x) d \mu_{h, k}(\gamma)
$$

for every $x \in G$ and $h, k \in \mathcal{H}$. ( $G$ is considered with the discrete topology, thus $\Gamma$ is compact.)

\section{MAIN RESULTS}

The following is the main result of the paper. Its proof is modeled after Artjomenko's proof of the Krein Extension Theorem ([2], also presented in [15]).

Theorem 2.1. Let $G_{1}$ be an ordered abelian group and $a \in G_{1}$. If $G_{2}$ is an abelian group, then every positive definite function $f:(-a, a) \times G_{2} \rightarrow \mathcal{L}(\mathcal{H})$ admits a positive definite extension to $G_{1} \times G_{2}$.

Proof. Let $G=G_{1} \times G_{2}$ and consider on $G$ the discrete topology, and let $\Gamma$ be the character group of $G$. Denote $V=(-a, a) \times G_{2}$ and let $\mathcal{P}(V)$ be the set of all functions $g: V \rightarrow \mathbf{C}$ with finite support. For $g \in \mathcal{P}(V)$ define

$$
\phi(x)= \begin{cases}g(x) k(x), & x \in V, \\ 0, & x \notin V .\end{cases}
$$


We prove that $\phi$ is positive definite on $G$ for every positive definite $g \in \mathcal{P}(V)$. Consider $x_{1}, x_{2}, \cdots, x_{n} \in G$. We have to prove that the operator matrix $\left(\phi\left(x_{i}-x_{j}\right)\right)_{i, j=1}^{n}$ is positive semidefinite. Without loss of generality, suppose that $x_{i}=\left(\lambda_{i}, \sigma_{i}\right)$, and $\lambda_{1} \leq \lambda_{2} \leq \cdots \leq \lambda_{n}$. Consider the undirected graph $H=(V, E)$ with vertex set $V=\{1,2, \cdots, n\}$ and edge set $E=\left\{(i, j) \mid i \neq j\right.$ and $\left.x_{j}-x_{i} \in V\right\}$. Then $H$ is a so-called proper interval graph (효). Define the partial operator matrix

$$
\left(A_{i j}\right)_{i, j=1}^{n}= \begin{cases}k\left(x_{i}-x_{j}\right) \text { for } & (i, j) \in E, \\ \text { unspecified for } & (i, j) \notin E .\end{cases}
$$

All fully specified principal submatrices of $A$ are positive semidefinite and $H$ is the graph associated with the pattern of $A$. By Corollary 3.2 in 1], $A$ admits a positive semidefinite extension $B=\left(B_{i j}\right)_{i, j=1}^{n}$. The matrix $\left(\phi\left(x_{i}-x_{j}\right)\right)_{i, j=1}^{n}$ is the Schur product of $B$ and $\left(g\left(x_{i}-x_{j}\right)\right)_{i, j=1}^{n}$ (all unspecified entries of $A$ correspond to zeros in $\left.\left(g\left(x_{i}-x_{j}\right)\right)_{i, j=1}^{n}\right)$. By a generalized version of Schur's Theorem ([12], proof of Theorem 4.3), it follows that $\left(\phi\left(x_{i}-x_{j}\right)\right)_{i, j=1}^{n}$ is positive semidefinite.

For $h \in \mathcal{H}$ define $\phi_{h}(x)=(\phi(x) h, h) ; \phi_{h}$ is a positive definite function on $G$ for every positive definite $g \in \mathcal{P}(V)$. Then

$$
\hat{\phi}_{h}(1)=\int_{G} \phi_{h}(x) d x=\sum_{x \in G} \phi_{h}(x) \geq 0,
$$

where the last inequality is a consequence of the fact that $\hat{\phi}_{h}$ is positive (see Theorem 1.9 .8 in 15]). Consequently, $\sum_{x \in G} g(x) k(x) \geq 0$, for every positive definite $g \in \mathcal{P}(V)$.

Define $T_{V}=\{\hat{g} \mid g \in \mathcal{P}(V)\}$ and let $T_{V}^{+}=\left\{p \in T_{V} \mid p \geq 0\right\}$. Every element of $T_{V}^{+}$is the Fourier transform of a positive definite function in $P(V)$. Define $l: T_{V} \rightarrow \mathcal{L}(\mathcal{H})$ by $l(q)=\sum_{x \in G} \check{q}(x) k(x)$. Then $l$ is a positive operator on $T_{V}$, which is an operator system in $C(\Gamma)$. We will prove that $l$ is completely positive.

Let $m>1$ and let $\mathcal{M}_{m}$ denote the set of all complex $m \times m$ matrices. Let $Z: G \rightarrow \mathcal{M}_{m}, Z(x)=\left(z_{i j}(x)\right)_{i, j=1}^{m}, z_{i j} \in \mathcal{P}(V)$, be a positive definite function. For $i, j=1, \cdots, m$, define

$$
\Phi_{i j}(x)= \begin{cases}z_{i j}(x) k(x), & x \in V, \\ 0, & x \notin V .\end{cases}
$$

We first prove that $\Phi(x)=\left(\Phi_{i j}(x)\right)_{i, j=1}^{m}$ is a positive definite matrix function on $G$. Let $x_{1}, x_{2}, \cdots, x_{n} \in G$. Without loss of generality, suppose that $x_{i}=\left(\lambda_{i}, \sigma_{i}\right)$, and $\lambda_{1} \leq \lambda_{2} \leq \cdots \leq \lambda_{n}$. Then,

$$
\left(\Phi\left(x_{k}-x_{l}\right)\right)_{k, l=1}^{n}=\left[Z\left(x_{k}-x_{l}\right)\right]_{k, l=1}^{n} \odot\left[B \otimes J_{m}\right],
$$

where $\odot$ denotes the Schur product, $B$ is a positive semidefinite extension of the partial matrix $A$ defined by (3), and $J_{m}$ is the $m \times m$ matrix with all entries equal to 1 . The matrix $\left[Z\left(x_{k}-x_{l}\right)\right]_{k, l=1}^{n}$ is positive semidefinite since $Z(x)$ is a positive definite function. Then (4) and the generalized version of Schur's Theorem ([12]) imply that $\left(\Phi\left(x_{k}-x_{l}\right)\right)_{k, l=1}^{n}$ is positive semidefinite, thus $\Phi(x)$ is a positive definite function.

Let $Q(x)=\left(q_{i j}(x)\right)_{i, j=1}^{m}$ be a matrix-valued function such that $q_{i j} \in T_{V}$ for every $i, j=1, \cdots, m$, and $Q(x) \geq 0$ for every $x \in G$. Then $\check{Q}(x)=\left(\check{q}_{i j}(x)\right)_{i, j=1}^{m}$ is a positive definite matrix function on $G$, which implies that the function $\left(\check{q}_{i j}(x) k(x)\right)_{i, j=1}^{m}$ 
is also positive definite. Let $\mathbf{h} \in \mathcal{H}_{n}=\bigoplus_{i=1}^{n} \mathcal{H}$. Define $\Phi_{\mathbf{h}}(x)=(\Phi(x) \mathbf{h}, \mathbf{h}) ; \Phi_{\mathbf{h}}$ is a positive definite (scalar) function on $G$. Then

$$
\hat{\Phi}_{\mathbf{h}}(1)=\int_{G} \Phi_{\mathbf{h}}(x) d x=\sum_{x \in G} \Phi_{\mathbf{h}}(x) \geq 0,
$$

the last inequality being a consequence of Theorem 1.9.8 in [15.

Relation (5) implies that the function $\mathbf{1}: T_{V} \otimes \mathcal{M}_{m} \rightarrow \mathcal{L}(\mathcal{H}) \otimes \mathcal{M}_{m}$, defined by $\mathbf{l}(Q)=\left(\sum_{x \in G} \breve{q}_{i j}(x) k(x)\right)_{i, j=1}^{m}$, is positive definite for every $m \geq 1$, which means that $l$ is completely positive.

Since $T_{V}$ is an operator system in $C(\Gamma)$, by Arveson's Theorem ([3], see also Theorem 6.5 in [11]), $l$ admits a (completely) positive extension $L: C(\Gamma) \rightarrow \mathcal{L}(\mathcal{H})$. By Theorem 1.1 there exists a semispectral family $\left\{\mu_{h, k}\right\}_{h, k \in \mathcal{H}}$ on $\Gamma$ such that

$$
(L(q) h, k)=\int_{\Gamma} q(\gamma) d \mu_{h, k}(\gamma)
$$

for every $q \in C(\Gamma)$ and $h, k \in \mathcal{H}$. Define $K: G \rightarrow \mathcal{L}(\mathcal{H})$ by

$$
(K(x) h, k)=\int_{\Gamma} \overline{\gamma(x)} d \mu_{h, k}(\gamma),
$$

for every $x \in G$ and $h, k \in \mathcal{H}$. Since $\left\{\mu_{h, k}\right\}_{h, k \in \mathcal{H}}$ is a semispectral family, Theorem 1.2 implies that $K$ is positive definite on $G$.

Let $x_{0} \in V$ and consider the function $\chi_{\left\{x_{0}\right\}} \in \mathcal{P}(V)$. For $h \in \mathcal{H}$, we have that

$$
\left(l\left(\hat{\chi}_{\left\{x_{0}\right\}}\right) h, h\right)=\sum_{x \in G}\left(\chi_{\left\{x_{0}\right\}} k(x) h, h\right)=\left(k\left(x_{0}\right) h, h\right) .
$$

Also,

$$
\hat{\chi}_{\left\{x_{0}\right\}}(\gamma)=\int_{G} \overline{\gamma(x)} \chi_{\left\{x_{0}\right\}} d x=\overline{\gamma\left(x_{0}\right)}
$$

Thus,

$$
\left(L\left(\hat{\chi}_{\left\{x_{0}\right\}}\right) h, h\right)=\int_{\Gamma} \hat{\chi}_{\left\{x_{0}\right\}}(\gamma) d \mu_{h}(\gamma)=\int_{\Gamma} \overline{\gamma\left(x_{0}\right)} d \mu_{h}(\gamma)=\left(K\left(x_{0}\right) h, h\right) .
$$

This implies that $\left(K\left(x_{0}\right) h, h\right)=\left(k\left(x_{0}\right) h, h\right)$ for every $h \in \mathcal{H}$, thus $K_{\mid(-a, a) \times G_{2}}=k$, and this completes the proof.

Corollary 2.2. Let $a$ be a positive real number and let $f:(-a, a) \rightarrow \mathcal{L}(\mathcal{H})$ be a positive definite function with a countable support. Then $f$ admits a positive definite extension to $\mathbf{R}$ which also has a countable support.

Proof. Apply Theorem 2.1 when $G_{1}$ is the additive group generated by the support of $f$ and $G_{2}$ the trivial group.

Corollary [2.2 can be viewed as a generalization of a result in [13] stating that every strictly positive definite matrix-valued almost periodic Wiener class function with spectrum in the real interval $(-a, a)$ can be extended to a (pointwise) strictly positive almost periodic function on $\mathbf{R}$ which also belongs to the Wiener class (see [16] for the scalar case).

The following result was proved in [7] for $r=2$ by a different approach. 
Corollary 2.3. Consider $\mathbf{Z}^{r}$ with the lexicographic order and $a \in \mathbf{Z}^{r}$. Then every positive definite function $f:(-a, a) \rightarrow \mathcal{L}(\mathcal{H})$ admits a positive definite extension to $\mathbf{Z}^{r}$.

Theorem 2.1 can be applied for a function defined on $(-a, a) \times \mathbf{Z}, a \in \mathbf{N}$. In this case, the function $f$ is assumed to be positive definite with respect to the set $[0, a) \times \mathbf{Z}$, which is different from the standard condition for $\mathbf{Z}^{2}$ and the lexicographic order, when positivity is considered with respect to the set

$$
\{(p, q): 0<p<a\} \cup\{(0, n): q \geq 0\} .
$$

Consider a function $f$ defined in the band $\left\{(p, q) \in \mathbf{Z}^{\mathbf{2}}:|p|<a\right\}$, positive definite with respect to the set (7). Let $f_{n}$ be the restriction of $f$ to the interval $((-a+1,-n),(a-1, n))$ and let $F_{n}$ be the extension of $f_{n}$ to $\mathbf{Z}^{2}$ given by Corollary 2.3. The sequence $\left\{F_{n}\right\}$ has a subsequence which converges pointwise in the weak topology to a function $F$ which is a positive extension of $f$. The above can be applied similarly for $\mathbf{Z}^{r}$. This leads to the following conclusion.

Corollary 2.4. Consider $\mathbf{Z}^{r}$ with the lexicographic order and let $a \in \mathbf{N}$. Let $S=$ $\left\{\left(m_{1}, m_{2}, \cdots, m_{r}\right) \in \mathbf{Z}^{r}:\left|m_{1}\right|<a\right\}$. Then every positive definite function $f: S \rightarrow$ $\mathcal{L}(\mathcal{H})$ admits a positive definite extension to $\mathbf{Z}^{r}$.

Let $\alpha_{1}, \alpha_{2}, \cdots, \alpha_{r} \geq 0$ be given. Consider in $\mathbf{Z}^{r}$ the set

$$
\begin{gathered}
P=\left\{\left(m_{1}, \cdots, m_{r}\right): \alpha_{1} m_{1}+\cdots+\alpha_{r} m_{r}>0\right\} \\
\cup\left\{\left(m_{1}, \cdots, m_{r}\right): \alpha_{1} m_{1}+\cdots+\alpha_{r} m_{r}=0, m_{1}=m_{2}=\cdots m_{k}=0, m_{k+1}>0\right\} .
\end{gathered}
$$

Then $P$ defines an order on $\mathbf{Z}^{r}$.

Corollary 2.5. Let $s>0$ and

$$
S=\left\{\left(m_{1}, \cdots, m_{r}\right) \in \mathbf{Z}^{r}:\left|\alpha_{1} m_{1}+\cdots+\alpha_{r} m_{r}\right| \leq s\right\},
$$

and let $k: S \rightarrow \mathcal{L}(\mathcal{H})$ be a positive definite function. Then $k$ admits a positive definite extension to $\mathbf{Z}^{r}$.

Proof. The result is a consequence of Theorem 2.1 combined with arguments such as those preceding Corollary 2.4

Corollary 2.5] can be viewed as a generalization of a result in 4 stating that every matrix-valued strictly positive definite function on a set of the form (9) for $r=2$ which belongs to the Wiener class can be extended to a (pointwise) positive function on $\mathbf{Z}^{2}$ which also belongs to the Wiener class.

\section{ACKNOWLEDGMENT}

The author thanks Professor Zoltán Sasvári for his suggestions and comments and Ramon Bruzual for pointing out an error in the previous version of the paper.

\section{REFERENCES}

[1] Gr. Arsene, Z. Ceauşescu, and T. Constantinescu, Schur analysis of some completion problems, Linear Algebra Appl., Vol. 109(1988), 1-35. MR 89k:47010

[2] A.P. Artjomenko, Hermitian positive functions and positive functionals, Dissertation, Odessa State University, 1941. Published in Teor. Funkcii, Funk. Anal. i Priložen, Vol. 41(1983), 1-16; Vol. 42(1984), 1-21 (Russian).

[3] W.B. Arveson, Subalegbras of $C^{*}$-algebras, Acta Math., 123(1969), 141-224. MR 40:6274 
[4] M. Bakonyi, L. Rodman, I. M. Spitkovsky, and H. J. Woerdeman, Positive matrix functions on the bitorus with prescribed Fourier coefficients in a band, J. Fourier Anal. Appl., Vol. 5(1999), 789-812. MR 2001c:42015

[5] Y.M. Berezansky and Y.G. Kondratiev, Spectral Methods in Infinite-Dimensional Analysis, Kluwer Academic Publishers, Dortrecht, 1995. MR 96d:46001a MR 96d:46001b

[6] Y.M. Berezansky and I.M. Gali, Positive definite functions of infinitely many variables on a layer, Ukrain. Mat. Zh., Vol. 24(1972), 435-464; English translation in Ukrain. Math. J. Vol. 24(1972).

[7] R. Bruzual and M. Dominguez, Extensions of operator valued positive definite functions on an interval of $\mathbf{Z}^{2}$ with the lexicographic order, Acta Sci. Math. Szeged, Vol. 66(2000), 623-631. CMP 2001:06

[8] M.C. Golumbic, Algorithmic Graph Theory and Perfect Graphs, Academic Press, New York, 1980. MR 81e:68081

[9] J. Friedrich and L. Klotz, On extensions of positive definite operator-valued function, Rep. Math. Phys., Vol. 26, No. 1(1988), 45-65. MR 90h:43005

[10] M.G. Krein, Sur le probléme de prolongement des functions hermitiniennes positives et continues, Dokl. Akad. Nauk. SSSR, Vol. 26(1940), 17-22.

[11] V. Paulsen, Completely Bounded Maps and Dilations, Pitman Research Notes in Mathematics, Vol. 146, New York, 1986. MR 88h:46111

[12] V.I. Paulsen, S.C. Power, and R.G. Smith, Schur products and matrix completions, J. Funct. Anal., Vol. 85(1989), 151-178. MR 90j:46051

[13] L. Rodman, I. Spitkovsky, and H.J. Woerdeman, Charathéodory-Toeplitz and Nehari problems for matrix valued almost periodic functions, Trans. Amer. Math. Soc., Vol. 350(1998), 2185-2227. MR 98h:47023

[14] W. Rudin, Fourier Analysis on Groups, Interscience Publishers, New York, 1962. MR 27:2808

[15] Z. Sasvári, Positive Definite and Definitizable Functions, Akademie Verlag, Berlin, 1994.

[16] I. Spitkovsky and H.J. Woerdeman, The Charathéodory-Fejér problem for almost periodic functions, J. Funct. Anal., Vol. 115(1993), 281-293. MR 94f:47020

[17] I. Suciu, Function Algebras, Editura Acamediei Române, Bucharest, 1975. MR 51:6428

Department of Mathematics, Georgia State University, Atlanta, Georgia 30303

E-mail address: mbakonyi@cs.gsu.edu 